\title{
Reactivity of Hydrazine, Some Hydrazine derivatives and Diamines toward Ethyl 2,2-Dicyano-1-aryl (or alkyl) vinylcarbamate
}

\author{
Fathi Zribi*, Mansour Salem and Fakher Chabchoub
}

Laboratoire de Chimie Appliquée : Hétérocycles, Corps gras et Polymères à la Faculté des Sciences de Sfax. Route de Soukra Km 3.5. BP 802. Sfax 3000. Tunisia

*Corresponding author: zfnfethi@yahoo.fr

\section{ABSTRACT}

A series of pyrimidinone and dipyrimidinone derivatives $2 \mathbf{a}-\mathbf{c}, \mathbf{4 a - e}$ and $\mathbf{6 a - f}$ has been synthesized via the reaction between ethyl 2,2-dicyano-1-aryl(or alkyl)vinylcarbamate derivatives 1a-d and hydrazine derivatives or diamines. The reactivity of compounds $\mathbf{1 a - d}$ toward hydrazine is studied. The result is the formation of triazolones 5a-d rather than pyrimidinone derivatives.

\section{Indexing terms/Keywords}

Hydrazine derivatives; ethyl 2,2-dicyano-1-aryl(or alkyl)vinylcarbamate; pyrimidinones; triazolones

\section{Council for Innovative Research}

Peer Review Research Publishing System

Journal: Journal of Advances in Chemistry

Vol. 10, No. 2

editorjaconline@gmail.com

www.cirjac.com 


\section{INTRODUCTION}

Over the years pyrimidinones derivatives have emerged as an interesting class of heterocycles with an astonishingly wide range of applications in pharmaceutical chemistry [1-4]. It is of great interest that specifically functionalized pyrimidinones may possess specific biological properties. Some are endowed with antiviral activity [5-6], antitumor activity [7-8], anti-inflammatory [9], antioxidant [10]. Many synthetic procedures exist for the synthesis of pyrimidinones derivatives [11-15]. However, the development of simple, easy and efficient methodologies to get pyrimidinones is one of the major aspects in organic synthesis. In fact, the ethyl 2,2-dicyano-1- aryl (or alkyl)vinylcarbamate derivatives are valuable intermediates in a variety of synthetic transformations. In the present study, we report the synthesis of some new pyrimidinones, dipyrimidinones and triazolones derivatives.

\section{RESULTS AND DISCUSSION}

Pyrimidine derivatives 2a-c were synthesized by cyclization, involving the reaction of substrates 1a-c with phenylhydrazine in chlorobenzene under reflux (scheme 1).

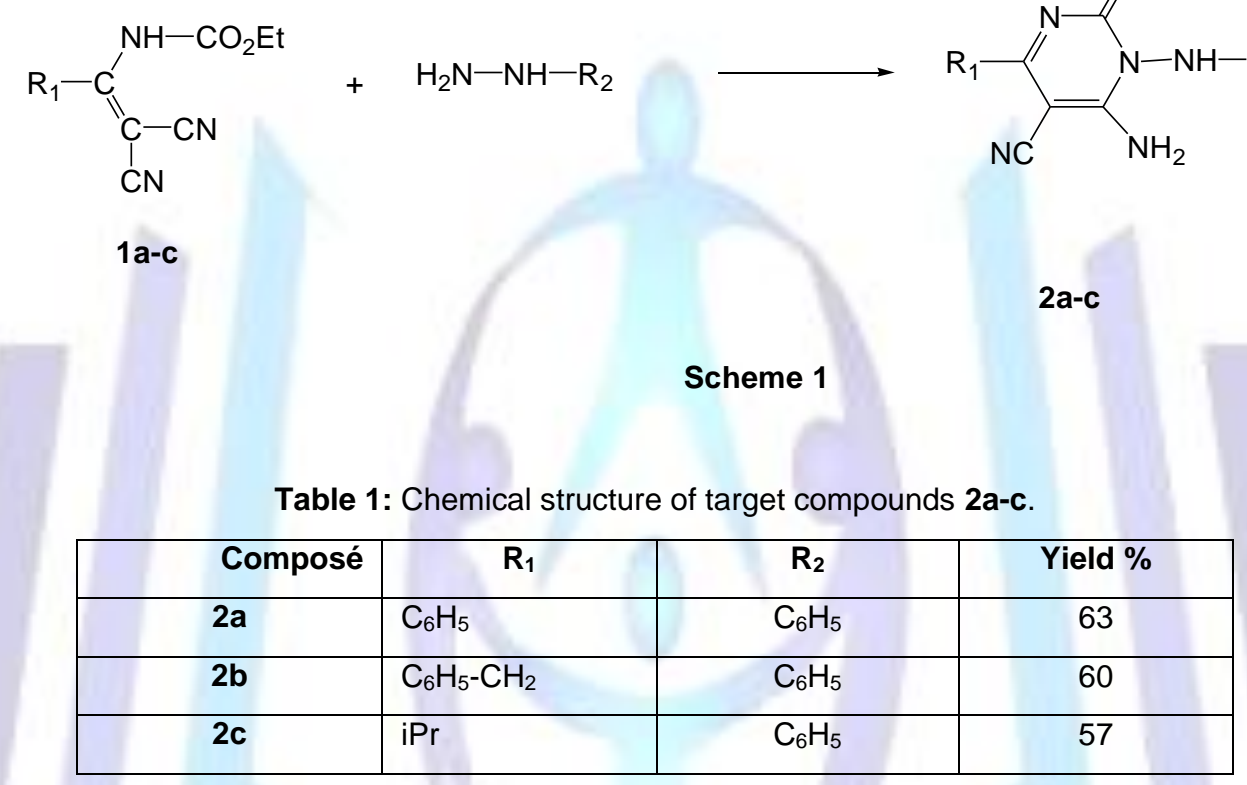

The prepared compounds were characterized by spectroscopic techniques. The IR spectra showed the presence of two $\mathrm{NH}$ groups stretching at $3420-3241 \mathrm{~cm}^{-1}$ and $\mathrm{CN}$ group stretching at $2220-2210 \mathrm{~cm}^{-1}$. In ${ }^{1} \mathrm{H}$ NMR spectra the most significant information was the disappearance of the triplet and the quadruplet of ethoxy groups of the starting reagent 1ac.

Compounds $\mathbf{2 a - c}$ can be used for the synthesis of heterocyclic compounds. For example we have condensed $\mathbf{2 b}$ with tetraethyl othocarbonate. The result is the formation of 7-benzyl-2-ethoxy-5-oxo-3-phenyl-3,5-dihydro$[1,2,4]$ triazolo[1,5-f]pyrimidine-8-carbonitrile 3 (scheme 2). Further work is in progress to obtain new structures by condensing compounds $2 \mathrm{a}-\mathrm{c}$ with orthoester, tetraethyl othocarbonate, carbon disulfide...<smiles>CCOc1nc2c(C#N)c(Cc3ccc(-c4ccccc4)cc3)nc(=O)n2n1-c1ccccc1</smiles>

$2 \mathbf{b}$

Scheme 2

Furthermore, the reactivity of $\mathbf{1}$ towards benzohydrazide and acetohydrazide was also investigated as an alternative route to obtain pyrimidinylbezamide derivatives $\mathbf{4 a - b}$ and pyrimidinylacetamide derivatives $\mathbf{4 c}$-e (scheme 3 ). The reaction was carried out in ethanol under reflux (benzohydrazide and acetohydrazide are not soluble in chlorobenzene). The intracyclization of compounds 4 a-e is not observed (scheme 3 ). 
<smiles>COC(=O)NC(Br)=C(C#N)C#N</smiles>

1a-c

Scheme 3<smiles>[R]C(=O)Nn1c(N)c(C#N)c([R])nc1=O</smiles>

4a-e

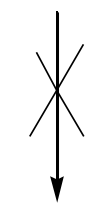<smiles>[R2]c1cc2c(C#N)c(Br)nc(=O)n2[nH]1</smiles>

Table 2: Chemical structure of target compounds 4a-e.

\begin{tabular}{|c|c|c|c|}
\hline Composé & $\mathbf{R}_{\mathbf{1}}$ & $\mathbf{R}_{\mathbf{2}}$ & Yield \% \\
\hline $\mathbf{4 a}$ & $\mathrm{C}_{6} \mathrm{H}_{5}$ & $\mathrm{C}_{6} \mathrm{H}_{5}$ & 68 \\
\hline $\mathbf{4 b}$ & $\mathrm{iPr}$ & $\mathrm{C}_{6} \mathrm{H}_{5}$ & 69 \\
\hline $\mathbf{4 c}$ & $\mathrm{C}_{6} \mathrm{H}_{5}$ & $\mathrm{CH}_{3}$ & 72 \\
\hline $\mathbf{4 d}$ & $\mathrm{C}_{6} \mathrm{H}_{5}-\mathrm{CH}_{2}$ & $\mathrm{CH}_{3}$ & 52 \\
\hline $\mathbf{4 e}$ & $\mathrm{iPr}$ & $\mathrm{CH}_{3}$ & 62 \\
\hline
\end{tabular}

The structures of compounds 4 a-e are in accordance with their spectroscopic data. These new products were assigned by IR, NMR and mass spectroscopy. The IR spectra showed bands $(\mathrm{NH}),(\mathrm{CN})$, and $(2 \mathrm{C}=\mathrm{O})$. In the ${ }^{1} \mathrm{HNMR}$ spectra of compounds $4 \mathrm{c}-\mathbf{e}$ we have noticed the appearance of the singlet of methyl groups at around $2 \mathrm{ppm}$.

In addition, heating 1a-d with hydrazine hydrate in chlorobenzene furnished trizol-3-one derivatives 5 a-d rather than pyrimidinone derivatives 5 'a-d (scheme 4).<smiles>CCOC(=O)NC(Br)=C(C#N)C#N</smiles>

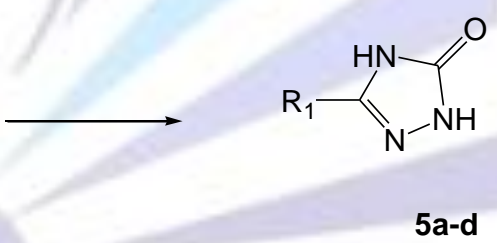

1a-d<smiles>CC(C)(C)C(C)(C)C</smiles><smiles>N#Cc1c(Br)nc(=O)n(N)c1N</smiles>

5'a-d

\section{Scheme 4}


The reaction products were confirmed on the basis of their spectral data and mass spectroscopy (5a-b). The IR spectra of the isolated products showed the absence of cyano groups. It also showed absorption bands due to (NH) and $(\mathrm{C}=\mathrm{O})$. Besides, the mass spectra displayed the respective $[\mathrm{M}+\mathrm{H}]^{+}$peaks.

Hydrazine hydrate reacts with its two nucleophilic centers $\left(\mathrm{NH}_{2}\right.$ groups) but hydrazine derivatives (phenylhydrazine, benzohydrazide and acetohydrazide) react with one of their nucleophilic centers $\left(\mathrm{NH}_{2}\right.$ and not $\left.\mathrm{NH}\right)$. This may be due to the conjugation of $\mathrm{NH}$ with carbonyl or phenyl groups.

To further explore the synthetic potential of enamine 1, the condensation of 1a-b with diamine (2,7-diam inofluorene, orthodianisidine and 1,4-benzenediamine) was investigated. Thus, refluxing ethyl 2,2-dicyano-1-arylvinylcarbamate derivatives 1a-b with diamine in chlorobenzene afforded dipyrimidinone derivatives 6a-f (scheme 5).

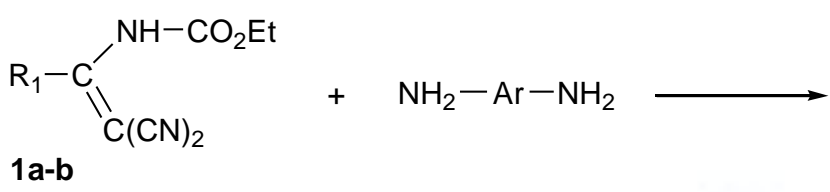<smiles>[R]c1nc(=O)n([Al]n2c(N)c(C#N)c([R])nc2=O)c(N)c1C#N</smiles>

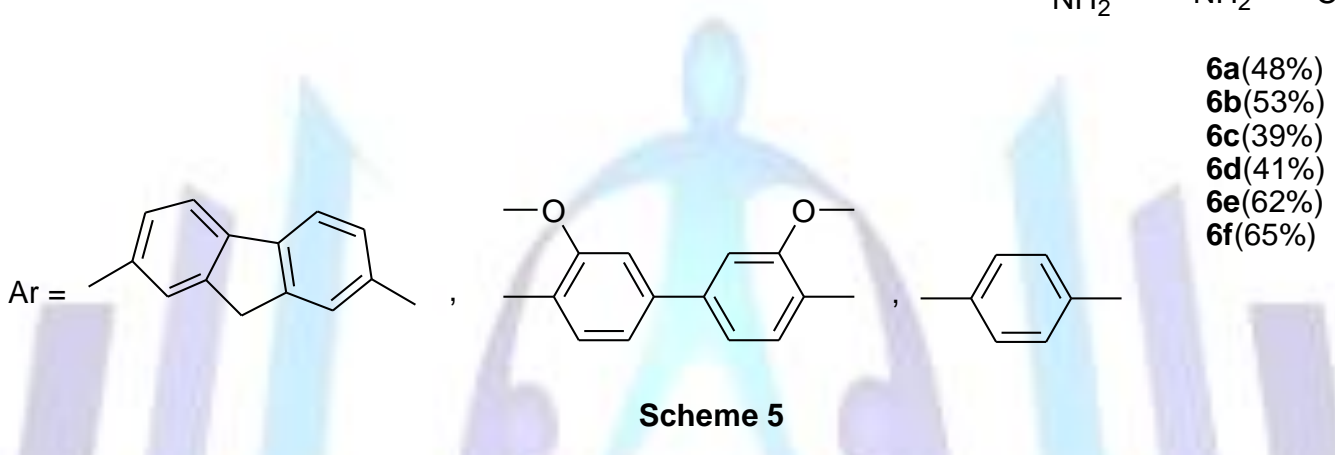

The structure of compounds 6a-f was proved on the basis of spectral data and mass spectroscopy. The IR spectra of 6a-f indicated the presence of $\mathrm{NH}$ group stretching at $3328-3132 \mathrm{~cm}^{-1}, \mathrm{CN}$ group stretching at $2207-2210 \mathrm{~cm}^{-1}$ and $\mathrm{C}=\mathrm{O}$ group stretching at $1660-1676 \mathrm{~cm}^{-1}$. The ${ }^{1} \mathrm{HNMR}$ spectra of compounds $6 \mathrm{a}-\mathbf{b}$ indicated a singlet at $\delta 3.74-3.79$ due to $\mathrm{CH}_{2}$ group. Also, the mass spectra showed the respective $[\mathrm{M}+\mathrm{H}]^{+}$peaks.

\section{CONCLUSION}

In conclusion, in this work we have tested the reactivity of ethyl 2,2-dicyano-1-aryl(or alkyl)vinylcarbamate derivatives toward phenylhydrazine, benzohydrazide, acetohydrazide, hydrazine and some diamine (2,7-diaminofluorene, orthodianisidine and 1,4-benzenediamine). Results demonstrate that hydrazine and its derivatives react differently to the compound 1a-d even though they have the same nucleophilic centre.

\section{EXPERIMENTAL}

Melting points are recorded in degrees Celsius on a Kofler apparatus. All reactions were followed by TLC (E. Merck Kieselgel 60 F-254), with UV detection at $254 \mathrm{~nm}$. The IR spectra were recorded in the solid state as $\mathrm{KBr}$ discs on a Perkin-Elmer PARAGON 1000 FT-IR spectrometer. ${ }^{1} \mathrm{H}$ and ${ }^{13} \mathrm{C}$ NMR were determined in solution in DMSO- $d_{6}$ with an AC Bruker spectrometer at $300 \mathrm{MHz}$ using TMS as an internal standard. The mass spectra were recorded on an ion trap mass spectrometer (Finnigan LCQ Deca XP Max) using electrospray as an ionization source. The purity of all compounds was determined by LC-PDA-MS methods and was found to be in the range between $96-99 \%$.

\section{GENERAL EXPERIMENTAL PROCEDURE}

\section{General procedure for the preparation of 2a-c , 4a-e and 6a-f.}

To a magnetically stirred solution of the ethyl 2,2-dicyanolvinylcarbamate derivatives $1 \mathrm{a}-\mathrm{d}$ (1 mmol) in chlorobenzene or ethanol $(10 \mathrm{~mL})$, the appropriate hydrazine derivatives or diamine $(1.2 \mathrm{mmol})$ were added and the reaction mixture stirred under reflux. The progress of the reaction was monitored by TLC (mobile phase, ethyl acetate: dichloromethane; $70 / 30 ; \mathrm{v} / \mathrm{v}$ ). The resulting mixture was allowed to cool at room temperature. The precipitate formed was isolated by filtration and washed with diethyl ether to obtain the pure product.

\section{General procedure for the preparation of 3}

A mixture of $\mathbf{2 b}(1 \mathrm{mmole})$, tetraethyl othocarbonate $(5 \mathrm{ml})$ and few drops of acetic acid was heated under reflux for $24 \mathrm{hr}$. The progress of the reaction was monitored by TLC (mobile phase, diethyl ether: hexane; 60/40;v/v). The solvent was removed in vacuo. Column chromatography purification using diethyl ether/ hexane as eluent gave the product 3. 


\section{Spectral Data of New Compounds}

6-amino-2-oxo-4-phenyl-1-(phenylamino)-1,2-dihydropyrimidine-5-carbonitrile $2 a$ : Yield $=63 \% ; \mathrm{mp}=212^{\circ} \mathrm{C} ; \mathrm{IR}$ $(\mathrm{KBr}) \mathrm{v}=3420-3319(\mathrm{NH}), 2236(\mathrm{CN}), 1694 \quad(\mathrm{C}=\mathrm{O}) \mathrm{cm}^{-1} ;{ }^{1} \mathrm{H}-\mathrm{NMR}$ (DMSO-d6): $\delta=6.88-7.99(\mathrm{~m}, 13 \mathrm{H}) ;{ }^{13} \mathrm{C}-\mathrm{NMR}$ (DMSO-d6): $\delta=72.8(\mathrm{C5}), 118.5$ (CN), 153.4(C2), 160.5(C4), 175.1(C6), 125.4-145.2(Carom).

6-amino-4-benzyl-2-oxo-1-(phenylamino)-1,2-dihydropyrimidine-5-carbonitrile $2 \mathrm{~b}: Y i e l d=60 \% \quad ; \mathrm{mp}=178^{\circ} \mathrm{C} ; \quad \mathrm{IR}$ $(\mathrm{KBr}) \mathrm{v}=3410-3241(\mathrm{NH}), 2220(\mathrm{CN}), 1674(\mathrm{C}=\mathrm{O}) \mathrm{cm}^{-1} ;{ }^{1} \mathrm{H}-\mathrm{NMR}(\mathrm{DMSO}-\mathrm{d} 6): \delta=3.86(\mathrm{~s}, 2 \mathrm{H}), 6.62-7.33(\mathrm{~m}, 10 \mathrm{H})$, $8.63\left(\mathrm{~s}, 2 \mathrm{H}, \mathrm{NH}_{2}\right), 8.76(\mathrm{~s}, 1 \mathrm{H}, \mathrm{NH}) ;{ }^{1} \mathrm{H}-\mathrm{NMR}$ (DMSO-d6): $43.4\left(\mathrm{CH}_{2}\right), 73.5(\mathrm{C} 5), 116.1(\mathrm{CN}), 153.0(\mathrm{C} 2), 160.4(\mathrm{C} 4)$, 174.9(C6), 121.3-146.2(C $\left.\mathrm{C}_{\text {arom }}\right)$.

6-amino-4-isopropyl-2-oxo-1-(phenylamino)-1,2-dihydropyrimidine-5-carbonitrile $2 c$ :Yield = 57\%; $\mathrm{mp}=148^{\circ} \mathrm{C} ; \quad \mathrm{IR}$ $(\mathrm{KBr}) \vee=3385-3275(\mathrm{NH}), 2212(\mathrm{CN}), 1690(\mathrm{C}=\mathrm{O}) \mathrm{cm}^{-1} ;{ }^{1} \mathrm{H}-\mathrm{NMR}(\mathrm{DMSO}-\mathrm{d} 6): \delta=1.07(\mathrm{~d}, 6 \mathrm{H}), 2.48(\mathrm{~m}, 1 \mathrm{H}), 6.63-$ $7.23(\mathrm{~m}, 5 \mathrm{H}), 8.52\left(\mathrm{~s}, 2 \mathrm{H}, \mathrm{NH}_{2}\right), 8.76(\mathrm{~s}, 1 \mathrm{H}, \mathrm{NH}) ;{ }^{3} \mathrm{C}-\mathrm{NMR}$ (DMSO-d6): $\delta=20.7\left(\mathrm{CH}_{3}\right), 34.9(\mathbf{C H}), 72.3(\mathrm{C} 5), 115.9$ $(\mathrm{CN}), 153.3(\mathrm{C} 2), 160.4(\mathrm{C} 4), 181.0(\mathrm{C} 6), 121.2-146.3\left(\mathrm{C}_{\text {arom }}\right)$.

7-benzyl-2-ethoxy-5-oxo-3-phenyl-3,5-dihydro-[1,2,4]triazolo[1,5-f]pyrimidine-8-carbonitrile 3: Yield = 20\%; IR $(\mathrm{KBr})$ $\mathrm{v}=2216(\mathrm{CN}), 1684(\mathrm{C}=\mathrm{O}) \mathrm{cm}^{-1}$; ${ }^{1} \mathrm{H}-\mathrm{NMR}$ (DMSO-d6): $\delta=0.93(\mathrm{t}, 3 \mathrm{H}), 3.62(\mathrm{~s}, 2 \mathrm{H}), 4.11(\mathrm{q}, 2 \mathrm{H}), 7.21-7.93(\mathrm{~m}, 10 \mathrm{H}) ;$ ${ }^{13}$ C-NMR (DMSO-d6): $\delta=13.9\left(\mathrm{CH}_{3}\right), 31.7\left(\mathrm{CH}_{2}\right), 36.7\left(\mathrm{CH}_{2}\right), 74.4(\mathrm{C} 8), 118.0(\mathrm{CN}), 138.3(\mathrm{C} 7), 147.2(\mathrm{C} 5), 153.0(\mathrm{C} 2)$, 124.0-129.6(C $\left.\mathrm{C}_{\text {arom}}\right)$.

N-(6-amino-5-cyano-2-oxo-4-phenylpyrimidin-1(2H)-yl)benzamide 4a: Yield $=68 \% ; \mathrm{mp}=256^{\circ} \mathrm{C} ; \mathrm{IR}(\mathrm{KBr}) \mathrm{v}=3361$ $3253(\mathrm{NH}), 2216(\mathrm{CN}), 1746(\mathrm{C}=\mathrm{O}), 1682(\mathrm{C}=\mathrm{O}) \mathrm{cm}^{-1} ;{ }^{1} \mathrm{H}-\mathrm{NMR}(\mathrm{DMSO}-\mathrm{d} 6): \delta=7.44-7.99(\mathrm{~m}, 10 \mathrm{H}), 9.2(\mathrm{~s}, 2 \mathrm{H}, \mathrm{NH})$, $10.60(\mathrm{~s}, 1 \mathrm{H}, \mathrm{NH}) ;{ }^{13} \mathrm{C}-\mathrm{NMR}$ (DMSO-d6): $\delta=72.5$ (C5), 118.5 (CN), 156.8(C2) , 160.7(C4), 166.9(C6), 171.8 (CO), $125.2-$ $137.1\left(\mathrm{C}_{\text {arom }}\right)$.

N-(6-amino-5-cyano-4-isopropyl-2-oxopyrimidin-1(2H)-yl)benzamide 4b: Yield $=69 \% ; \mathrm{mp}=196^{\circ} \mathrm{C} ; \mathrm{IR}(\mathrm{KBr}) \mathrm{v}=3348$ $3221(\mathrm{NH}), 2212(\mathrm{CN}), 1733(\mathrm{C}=\mathrm{O}), 1681(\mathrm{C}=\mathrm{O}) \mathrm{cm}^{-1}$; ${ }^{1} \mathrm{H}-\mathrm{NMR}$ (DMSO-d6): $\delta=1.14(\mathrm{~d}, 6 \mathrm{H}), 3.01(\mathrm{~m}, 1 \mathrm{H}), 7.39-7.97$ $(\mathrm{m}, 5 \mathrm{H}), 8.5\left(\mathrm{~s}, 2 \mathrm{H}, \mathrm{NH}_{2}\right), 11.11(\mathrm{~s}, 1 \mathrm{H}, \mathrm{NH}) ;{ }^{13} \mathrm{C}-\mathrm{NMR}$ (DMSO-d6): $\delta=20.8\left(\mathrm{CH}_{3}\right), 35.0(\mathbf{C H}), 72.3(\mathrm{C} 5), 115.7(\mathbf{C N})$, 152.4(C2) , 159.9 (C4), 166.9(C6), 181.5 (CO), 127.5-133.0( $\left.\mathrm{C}_{\text {arom }}\right)$.

N-(6-amino-5-cyano-2-oxo-4-phenylpyrimidin-1(2H)-yl)acetamide 4c: Yield $=72 \% ; \mathrm{mp}=248{ }^{\circ} \mathrm{C} ; \mathrm{IR}(\mathrm{KBr}) \mathrm{v}=3373-$ $3249(\mathrm{NH}), 2213(\mathrm{CN}), 1724(\mathrm{C}=\mathrm{O}), 1688(\mathrm{C}=\mathrm{O}) \mathrm{cm}^{-1}$; ${ }^{1} \mathrm{H}-\mathrm{NMR}$ (DMSO-d6): $\delta=2.03(\mathrm{~s}, 3 \mathrm{H}), 7.50-7.76(\mathrm{~m}, 5 \mathrm{H}), 8.65$ $\left(\mathrm{s}, 2 \mathrm{H}, \mathrm{NH}_{2}\right.$ ), 10.73 (s, $\left.1 \mathrm{H}, \mathrm{NH}\right) ;{ }^{13} \mathrm{C}-\mathrm{NMR}$ (DMSO-d6): $\delta=72.4$ (C5), 116.5 (CN), 151.9(C2), 160.4 (C4), 168.5(C6), 171.7 (CO), 128.7-137.2 ( $\left.\mathrm{C}_{\text {arom}}\right)$.

N-(6-amino-4-benzyl-5-cyano-2-oxopyrimidin-1(2H)-yl)acetamide 4d: Yield $=52 \% ; \mathrm{mp}=226^{\circ} \mathrm{C} ; \mathrm{IR}(\mathrm{KBr}) \mathrm{v}=3491$ $3224(\mathrm{NH}), 2226(\mathrm{CN}), 1735(\mathrm{C}=\mathrm{O}), 1687(\mathrm{C}=\mathrm{O}) \mathrm{cm}^{-1}$; ${ }_{1}^{1} \mathrm{H}-\mathrm{NMR}$ (DMSO-d6): $\delta=1.98(\mathrm{~s}, 3 \mathrm{H}), 3.82(\mathrm{~s}, 2 \mathrm{H}), 7.24-7.30(\mathrm{~m}$, $5 \mathrm{H}), 8.58\left(\mathrm{~s}, 2 \mathrm{H}, \mathrm{NH}_{2}\right), 10.60(\mathrm{~s}, 1 \mathrm{H}, \mathrm{NH}) ;{ }^{13} \mathrm{C}-\mathrm{NMR}$ (DMSO-d6): $\delta=43.4\left(\mathrm{CH}_{2}\right), 20.9\left(\mathbf{C H}_{3}\right), 72.2(\mathrm{C} 5), 116.0(\mathrm{CN})$, 152.5(C2) , 159.7 (C4), 168.4(C6), 170.2 (CO), 127.3-136.9 (C $\left.\mathrm{C}_{\text {arom }}\right)$.

N-(6-amino-5-cyano-4-isopropyl-2-oxopyrimidin-1(2H)-yl)acetamide 4 e : Yield $=62 \% ; \mathrm{mp}=240^{\circ} \mathrm{C} ; \mathrm{IR}(\mathrm{KBr}) \mathrm{v}=3370$ $3230(\mathrm{NH}), 2231(\mathrm{CN}), 1745(\mathrm{C}=\mathrm{O}), 1688(\mathrm{C}=\mathrm{O}) \mathrm{cm}^{-1}$; ${ }^{\mathrm{H}-N M R}$ (DMSO-d6): $\delta=1.11(\mathrm{~d}, 6 \mathrm{H}), 1.99(\mathrm{~s}, 3 \mathrm{H}), 2.96(\mathrm{~m}, 1 \mathrm{H}$ ), 8.49 (s, 2H, NH$\left.{ }_{2}\right), 10.58(\mathrm{~s}, 1 \mathrm{H}, \mathrm{NH}) ;{ }^{13} \mathrm{C}-\mathrm{NMR}$ (DMSO-d6): $\delta=20.8\left(\mathrm{CH}_{3}\right), \quad 34.9(\mathbf{C H}), \mathrm{C}_{7} 20.9\left(\mathrm{CH}_{3}\right), 72.2(\mathrm{C} 5), 115.7$ $(\mathrm{CN}), 152.3(\mathrm{C} 2)$, $158.5(\mathrm{C} 4), 159.7(\mathrm{C} 6), 170.2(\mathrm{CO}), 127.5-133.0\left(\mathrm{C}_{\text {arom }}\right)$.

5-phenyl-2H-1,2,4-triazol-3(4H)-one 5a: Yield $=55 \% ; \mathrm{mp}=276^{\circ} \mathrm{C} ; \mathrm{IR}(\mathrm{KBr}) \mathrm{v}=3450-3189(\mathrm{NH}), 1748(\mathrm{C}=\mathrm{O}) \mathrm{cm}^{-1}$ ${ }^{1} \mathrm{H}-\mathrm{NMR}$ (DMSO-d6): $\delta=7.43-7.75(\mathrm{~m}, 5 \mathrm{H}), 11.69(\mathrm{~s}, 2 \mathrm{H}, 2 \mathrm{NH}) ;{ }^{13} \mathrm{C}-\mathrm{NMR}$ (DMSO-d6): $\delta=145.5$ (C5), $156.8(\mathrm{C} 3)$, 125.2-130.3 (Carom); MS-(+)ESI: $m / z(\%): 162\left([\mathrm{M}+\mathrm{H}]^{+}, 100\right)$.

5-p-tolyl-2H-1,2,4-triazol-3(4H)-one 5b: Yield =60\%, mp= 269 ${ }^{\circ} \mathrm{C} ; \mathrm{IR}(\mathrm{KBr}) \mathrm{v}=3438-3180(\mathrm{NH}), 1713(\mathrm{C}=\mathrm{O}) ;{ }^{1} \mathrm{H}-\mathrm{NMR}$ : (DMSO-d6): $\delta=2.34\left(\mathrm{~s}, 3 \mathrm{H}, \mathrm{CH}_{3}\right), 7.30$ ( d, 2H, J=6.0), 7.70( d, 2H, J=6.0), $11.65(\mathrm{~s}, 2 \mathrm{H}, 2 \mathrm{NH}) ;{ }^{13} \mathrm{C}-\mathrm{NMR}$ (DMSO-d6): $\delta=$ $20.8\left(\mathrm{CH}_{3}\right), 145.1$ (C5), 156.3 (C3), 124.3-139.3(C arom); MS-(+)ESI: $\mathrm{m} / \mathrm{z}(\%): 190\left([\mathrm{M}+\mathrm{H}]^{+}, 100\right)$

5-benzyl-2H-1,2,4-triazol-3(4H)-one 5c: Yield $=44 \% ; m p=212^{\circ} \mathrm{C} ; \mathrm{IR}(\mathrm{KBr}) \mathrm{v}=3453-3189(\mathrm{NH}), 1767(\mathrm{C}=\mathrm{O}) \mathrm{cm}^{-1} ;{ }^{1} \mathrm{H}-$ NMR (DMSO-d6) : $\delta=3.70(\mathrm{~s}, 2 \mathrm{H}), 7.21-7.32(\mathrm{~m}, 5 \mathrm{H}), 11.21(\mathrm{~s}, 2 \mathrm{H}, 2 \mathrm{NH}) ;{ }^{13} \mathrm{C}-\mathrm{NMR}(\mathrm{DMSO}-\mathrm{d} 6): \delta=32.9\left(\mathrm{CH}_{2}\right), 146.7$ (C5), 156.7 (C3), 127.2-136.7 ( $\left.\mathrm{C}_{\text {arom }}\right)$.

5-isopropyl-2H-1,2,4-triazol-3(4H)-one 5d: Yield = 48\%; $\mathrm{mp}=220^{\circ} \mathrm{C} ; \mathrm{IR}(\mathrm{KBr}) \mathrm{v}=3382-3181(\mathrm{NH}), 1733(\mathrm{C}=\mathrm{O}) \mathrm{cm}{ }^{-1}$; ${ }^{1} \mathrm{H}-\mathrm{NMR}(\mathrm{DMSO}-\mathrm{d} 6): \delta=1.13(\mathrm{~d}, 6 \mathrm{H}), 2.94(\mathrm{~m}, 1 \mathrm{H}), 11.32(\mathrm{~s}, 2 \mathrm{H}, 2 \mathrm{NH}),{ }^{13} \mathrm{C}-\mathrm{NMR}(\mathrm{DMSO}-\mathrm{d} 6): \delta=20.7\left(\mathrm{CH}_{3}\right)$, $35.0(\mathrm{CH}), 146.3(\mathrm{C} 5), 156.8(\mathrm{C} 3)$.

6-amino-1-(7-(6-amino-5-cyano-2-oxo-4-phenylpyrimidin-1(2H)-yl)-9H-fluoren-2-yl)-2-oxo-4-phenyl-1,2dihydropyrimidine-5-carbonitrile 6a: yield = 48\%; $\mathrm{C}_{35} \mathrm{H}_{22} \mathrm{~N}_{8} \mathrm{O}_{2}, \mathrm{M}=586 \mathrm{~g} \cdot \mathrm{mol}^{-1}, \mathrm{mp}=294^{\circ} \mathrm{C}$; IR $(\mathrm{KBr}) \mathrm{v}$ : 3315-3197 (NH2), $2208(\mathrm{CN}), 1676(\mathrm{C}=\mathrm{O}) \mathrm{cm}^{-1} ;{ }^{1} \mathrm{H}-\mathrm{NMR}$ : (DMSO-d6): $\delta=3.79\left(\mathrm{~s}, 2 \mathrm{H}, \mathrm{CH}_{2}\right), 6.62-7.84(\mathrm{~m}, 2 \mathrm{H}, \mathrm{Ar}-\mathrm{H}+2 \mathrm{NH}) ;{ }^{13} \mathrm{C}-$ NMR (DMSO-d6): $\delta=36.8\left(\mathrm{CH}_{2}\right), 72.7(\mathrm{C} 5), 117.1(\mathrm{CN}), 154.3(\mathrm{C} 6), 160.8(\mathrm{C} 2), 171.7(\mathrm{C} 4), 119.5-145.6\left(\mathrm{C}_{\text {arom}}\right)$; MS(+)ESI: $m / z(\%): 587\left([\mathrm{M}+\mathrm{H}]^{+}, 100\right)$.

6-amino-1-(7-(6-amino-5-cyano-2-oxo-4-p-tolylpyrimidin-1(2H)-yl)-9H-fluoren-2-yl)-2-oxo-4-p-tolyl-1,2-

dihydropyrimidine-5-carbonitrile $6 \mathrm{~b}$ : Yield $=53 \% ; \mathrm{C}_{37} \mathrm{H}_{26} \mathrm{~N}_{8} \mathrm{O}_{2}, \mathrm{M}=614 \mathrm{~g} \cdot \mathrm{mol}^{-1}, \mathrm{mp}=209^{\circ} \mathrm{C} ; \mathrm{IR}(\mathrm{KBr}) \mathrm{v}$ : $3327-3132$ (NH2), $2209(\mathrm{CN}), 1660(\mathrm{C}=\mathrm{O}) \mathrm{cm}^{-1}$; ${ }^{1} \mathrm{H}-\mathrm{NMR}$ : (DMSO-d6): $\delta=2.36\left(\mathrm{~s}, 6 \mathrm{H}, 2 \mathrm{CH}_{3}\right), 3.74\left(\mathrm{~s}, 2 \mathrm{H}, \mathrm{CH}_{2}\right), 6.62-7.90(\mathrm{~m}, 18 \mathrm{H}$, $\left.\mathrm{Ar}-\mathrm{H}+2 \mathrm{NH}_{2}\right) ;{ }^{13} \mathrm{C}-\mathrm{NMR}(\mathrm{DMSO}-\mathrm{d} 6): \delta=21.5\left(\mathrm{CH}_{3}\right), 36.8\left(\mathrm{CH}_{2}\right), 72.6(\mathrm{C} 5), 117.2(\mathrm{CN}), 154.3(\mathrm{C} 6), 160.8(\mathrm{C} 2), 171.4$

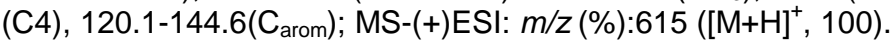


Compound 6c: Yield = 39\%; $\mathrm{C}_{36} \mathrm{H}_{26} \mathrm{~N}_{8} \mathrm{O}_{4}, \mathrm{M}=634 \mathrm{~g} \cdot \mathrm{mol}^{-1}, \mathrm{mp}=270^{\circ} \mathrm{C} ; \mathrm{IR}(\mathrm{KBr})$ v: 3319-3196 (NH2), $2206(\mathrm{CN}), 1673$ $(\mathrm{C}=\mathrm{O}) \mathrm{cm}^{-1}$; ${ }^{1} \mathrm{H}-\mathrm{NMR}$ : (DMSO-d6): $\delta=3.95\left(\mathrm{~s}, 6 \mathrm{H}, 2 \mathrm{O}-\mathrm{CH}_{3}\right), 7.33-8.35\left(\mathrm{~m}, 2 \mathrm{H}, \mathrm{Ar}-\mathrm{H}+2 \mathrm{NH}_{2}\right) ;{ }^{13} \mathrm{C}-\mathrm{NMR}$ (DMSO-d6): $\delta=$ $56.0\left(\mathrm{O}-\mathrm{CH}_{3}\right), 72.0$ (C5), 117.1 (CN), 154.8 (C6), 159.9 (C2), 171.9 (C4), 119.5-142.9(Carom); MS-(+)ESI: m/z (\%):635 $\left([\mathrm{M}+\mathrm{H}]^{+}, 100\right)$.

Compound 6d: Yield $=41 \% ; \mathrm{C}_{38} \mathrm{H}_{30} \mathrm{~N}_{8} \mathrm{O}_{4}, \mathrm{M}=662 \mathrm{~g} \cdot \mathrm{mol}^{-1}, \mathrm{mp}=265^{\circ} \mathrm{C}$; IR $(\mathrm{KBr}) \mathrm{v}: 3328-3200\left(\mathrm{NH}_{2}\right), 2210(\mathrm{CN}), 1666$ $(\mathrm{C}=\mathrm{O}) \mathrm{cm}^{-1}$; ${ }^{1} \mathrm{H}-\mathrm{NMR}:(\mathrm{DMSO}-\mathrm{d} 6): \delta=2.41\left(\mathrm{~s}, 6 \mathrm{H}, 2 \mathrm{CH}_{3}\right), 4.02\left(\mathrm{~s}, 6 \mathrm{H}, 2 \mathrm{O}-\mathrm{CH}_{3}\right), 7.35-7.79(\mathrm{~m}, 18 \mathrm{H}, \mathrm{Ar}-\mathrm{H}+2 \mathrm{NH}) ;{ }^{13} \mathrm{C}-$ NMR (DMSO-d6): $\delta=21.5\left(\mathrm{CH}_{3}\right), 56.5\left(\mathrm{O}-\mathrm{CH}_{3}\right), 72.1(\mathrm{C} 5), 117.1(\mathrm{CN}), 155.5(\mathrm{C} 6), 160.5(\mathrm{C} 2), 171.9(\mathrm{C} 4), 119.6-$ 143.3(C

6-amino-1-(4-(6-amino-5-cyano-2-oxo-4-phenylpyrimidin-1(2H)-yl)phenyl)-2-oxo-4-phenyl-1,2-dihydro pyrimidine-5carbonitrile 6e: Yield $=62 \% ; \mathrm{C}_{28} \mathrm{H}_{18} \mathrm{~N}_{8} \mathrm{O}_{2}, \mathrm{M}=498 \mathrm{~g} \cdot \mathrm{mol}^{-1}, \mathrm{mp}=255^{\circ} \mathrm{C}$; IR (KBr) v: 3312-3212 (NH2), $2208(\mathrm{CN}), 1673$ $(\mathrm{C}=\mathrm{O}) \mathrm{cm}^{-1}$; ${ }^{1} \mathrm{H}-\mathrm{NMR}$ : (DMSO-d6): $\delta=6.49-7.95\left(\mathrm{~m}, 18 \mathrm{H}, \mathrm{Ar}-\mathrm{H}+2 \mathrm{NH}_{2}\right) ;{ }^{13} \mathrm{C}-\mathrm{NMR}(\mathrm{DMSO}-\mathrm{d} 6): \delta=72.8(\mathrm{C} 5), 117.1$ (CN), 154.5 (C6), 160.9 (C2), 171.3 (C4), 120.9-137.4(Carom); MS-(+)ESI: $\mathrm{m} / \mathrm{z}(\%): 499\left([\mathrm{M}+\mathrm{H}]^{+}, 100\right)$.

6-amino-1-(4-(6-amino-5-cyano-2-oxo-4-p-tolylpyrimidin-1(2H)-yl)phenyl)-2-oxo-4-p-tolyl-1,2-dihydro pyrimidine-5carbonitrile 6f: Yield $=65 \% ; \mathrm{C}_{30} \mathrm{H}_{22} \mathrm{~N}_{8} \mathrm{O}_{2}, \mathrm{M}=526 \mathrm{~g} \cdot \mathrm{mol}^{-1}, \mathrm{mp}=270^{\circ} \mathrm{C} ; \mathrm{IR}(\mathrm{KBr}) \mathrm{v}: 3314-3207$ (NH2), 2207 (CN), 1674 $(\mathrm{C}=\mathrm{O}) \mathrm{cm}^{-1}$; ${ }^{1} \mathrm{H}-\mathrm{NMR}:(\mathrm{DMSO}-\mathrm{d} 6): \delta=2.39\left(\mathrm{~s}, 6 \mathrm{H}, 2 \mathrm{CH}_{3}\right), 6.66-7.95\left(\mathrm{~m}, 16 \mathrm{H}, \mathrm{Ar}-\mathrm{H}+2 \mathrm{NH}_{2}\right) ;{ }^{13} \mathrm{C}-\mathrm{NMR}(\mathrm{DMSO}-\mathrm{d} 6): \delta=$ $21.5\left(\mathrm{CH}_{3}\right), 73.3$ (C5), 117.26 (CN), 154.5 (C6), 161.0 (C2), 171.2 (C4), 122.1-141.4(Carom); MS-(+)ESI: m/z (\%):527 $\left([\mathrm{M}+\mathrm{H}]^{+}, 100\right)$.

\section{ACKNOWLEDGMENTS}

Thanks are due to the Ministry of Higher Education and Scientific Research and Technology in Tunisia and Sfax University for financial support

\section{REFERENCES}

[1] C. M. Wright, R. J. Chovatiya, N. E. Jameson, D. M. Turner, G. Zhu, S. Werner, D. M. Huryn, J. M. Pipas, B. W. Day, P. Wipf, J. L. Brodsky, Bioorg. Med. Chem. 16 (2008) 3291.

[2] S. W. Fewell, C. M. Smith, M. A. Lyon, T. P. Dumitrescu, P. Day, B. W. Wipf, J. L. Brodsky, J. Biol. Chem. 279 (2004) 51131.

[3] S. W. Fewell, B. W. Day, J. L. Brodsky, J. Biol. Chem. 276 (2001) 910.

[4] A. Rodina, M. Vilenchik, K. Moulick, J. Aguirre, J. Kim, A. Chiang, J. Litz, C. C. Clement, Y. Kang, Y. She, N. Wu, S. Felts, P. Wipf, J. Massague, X. Jiang, J. L. Brodsky, G. W. Krystal, G. Chiosis, Nat. Chem. Biol. 3 (2007) $498-507$.

[5] K. Singh, K. Singh, J. Balzarini, European Journal of Medicinal Chemistry 67 (2013) 428-433.

[6 ] S. F. Mohamed, E. M. Flefel, A. E. Amra, D. N. Abd El-Shafy, Eur. J. Med. Chem. 45 (2010) 1494-1501.

[7] P. Singh, P. Kaur, V. Luxami, S. Kaur, S. Kumar, Bioorg. Med. Chem. 15 (2007) 2386-2395.

[8] A. A. Abu-Hashem, M. M. Youssef, H. A. R. Hussein, J. Chin. Chem. Soc. 58 (2011) 41-48.

[9] B. Tozkoparan, M. Ertan, P. Kelicen, R. Demirdamar, II Farmaco 54 (1999) 588-593.

[10] S. Maddila, G. L. V. Damu, E. O. Oseghe, O. A. Abafe, C. Venakata Rao, P. Lavanya, J.Korean Chem. Soc. 56 (2012) 334-340.

[11] A. Chaker, F. Zribi, F Nepveu, F. Chabchoub, Chinese Chemical Letters (2014).

[12] G. Koza, S. Özca, E. Sahin, M. Balci, Tetrahedron 65 (2009) 5973-5976.

[13] P. Singh, A. Marwaha, H. Singh, M. P. Mahajan, Tetrahedron 61 (2005) 11999-12005.

[14] J. Safari, S. Ravandi, Journal of Molecular Catalysis A: Chemical 373 (2013) 72-77.

[15] H. Zhang, Z. Zhou, Z. Yao, F. Xu, Q Shen, Tetrahedron Letters, 50(2009)1622-1624. 